This is the accepted version of the following article:

Zor E., Morales-Narváez E., Alpaydin S., Bingol H., Ersoz M., Merkoçi A.. Graphene-based hybrid for enantioselective sensing applications. Biosensors and Bioelectronics, (2017). 87. : 410 - . 10.1016/j.bios.2016.08.074,

which has been published in final form at https://dx.doi.org/10.1016/j.bios.2016.08.074 (c) https://dx.doi.org/10.1016/j.bios.2016.08.074. This manuscript version is made available under the CC-BY-NC-ND 4.0 license http://creativecommons.org/licenses/by-nc-nd/4.0/ 


\section{Graphene-based hybrid for enantioselective sensing applications}

Erhan Zor ${ }^{\mathrm{a}, \mathrm{b}, \mathrm{c}}$, Eden Morales-Narváez ${ }^{\mathrm{a}}$, Sabri Alpaydin ${ }^{\mathrm{d}}$, Haluk Bingold ${ }^{\mathrm{d}}$ Mustafa Ersoz $^{\mathrm{e} *}$ and Arben Merkoçi $i^{\mathrm{a}, \mathrm{f} *}$

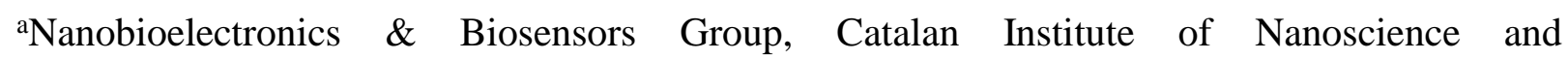
Nanotechnology (ICN2), CSIC and The Barcelona Institute of Science and Technology, Campus UAB, Bellaterra, Barcelona, 08193, Spain

${ }^{\mathrm{b}}$ Graduate School of Natural Sciences, Selcuk University, Konya, 42030, Turkey

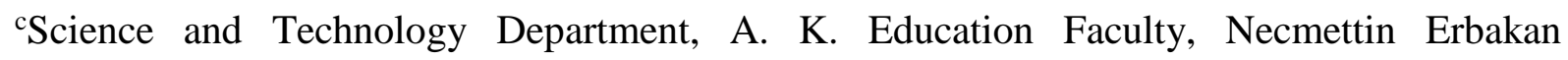
University, Konya, 42090, Turkey

dDepartment of Chemistry, A. K. Education Faculty, Necmettin Erbakan University, Konya, 42090, Turkey

${ }^{\mathrm{e} A d v a n c e d ~ T e c h n o l o g y ~ R e s e a r c h ~ a n d ~ A p p l i c a t i o n ~ C e n t e r, ~ S e l c u k ~ U n i v e r s i t y, ~ K o n y a, ~ 42030, ~}$ Turkey

fICREA, Pg. Lluís Companys 23, 08010 Barcelona, Spain.

*Corresponding authors:

Mustafa Ersoz ; E-mail: ersozm@gmail.com; Tel: +90332-2412484; Fax:+ 90332-2412499

Arben Merkoçi; E-mail: arben.merkoci@ icn2.cat; Tel: +34937374604; Fax:+34935868020

\section{Abstract}

Chirality is a major field of research of chemical biology and is essential in pharmacology. Accordingly, approaches for distinguishing between different chiral forms of a compound are of great interest. We report on an efficient and generic enantioselective sensor that is achieved 
by coupling reduced graphene oxide with $\gamma$-cyclodextrin $(r G O / \gamma-C D)$. The enantioselective sensing capability of the resulting structure was operated in both electrical and optical mode for of tryptophan enantiomers $(D-/ L$-Trp). In this sense, voltammetric and photoluminescence measurements were conducted and the experimental results were compared to molecular docking method. We gain insight into the occurring recognition mechanism with selectivity toward $D$ - and $L$-Trp as shown in voltammetric, photoluminescence and molecular docking responses. As an enantioselective solid phase on an electrochemical transducer, thanks to the different dimensional interaction of enantiomers with hybrid material, a discrepancy occurs in the Gibbs free energy leading to a difference in oxidation peak potential as observed in electrochemical measurements. The optical sensing principle is based on the energy transfer phenomenon that occurs between photoexcited $D$-/L-Trp enantiomers and $r G O / \gamma-C D$ giving rise to an enantioselective photoluminescence quenching due to the tendency of chiral enantiomers to form complexes with $\gamma-C D$ in different molecular orientations as demonstrated by molecular docking studies. The approach, which is the first demonstration of applicability of molecular docking to show both enantioselective electrochemical and photoluminescence quenching capabilities of a graphene-related hybrid material, is truly new and may have broad interest in combination of experimental and computational methods for enantiosensing of chiral molecules.

Keywords: Graphene oxide, cyclodextrin, enantioselectivity, sensor, molecular docking

\section{Introduction}

Chirality is a prominent characteristic of natural systems, and artificial enantioselective sensing and discrimination of biologically important chiral species is a crucial challenge in various applications related to sensors, catalysis, biomedicine and 
biotechnology (Wattanakit et al., 2014). During the past decade, chiral discrimination has become of increasing importance and many efforts have been devoted to the goal of understanding biochemical processes in biological phenomena involving artificial systems (Zor et al., 2013). Great success has been attained in the development of specific chiral selectors that enable enantioselective discrimination by high performance liquid chromatography (Bobbitt and Linder, 2001), NMR (Ema et al., 2007), chemiluminescence (Wang et al., 2008) and circular dichroism (Cao et al., 2013) techniques. Apart from the conventional application of these techniques, in recent years, electrochemical sensors have been frequently reported for discriminative sensing of bioactive compounds due to their advantages such as high stability and sensitivity, and they are easy to be integrated in various sensing platforms for advanced intelligent sensing (Trojanowicz, 2014).

The key step to produce an electrochemical chiral sensor is to build molecular architectures consisting of enantioselective sensing sites which exhibits different binding affinity for enantiomers (Kaminker et al., 2013). In addition to their host-guest properties with interest in sensing (Alarcón-Angeles et al., 2008) cyclodextrins (CDs) have been widely used for enantioselective extraction of bioactive compounds, various methods using these cyclic polysaccharides composed of glucose molecules have been reported as electrochemical chiral biosensors (Guo et al., 2010; Zor et al., 2015). However, the requirement for an electrochemical chiral sensor for discrimination of enantiomers is not only recognition of enantiomers but also a material enhancing and distinguishing the analytical signal (ex. electrical current) resulted by the interaction on the electrode surface. The unification of enantioselectivity with electrical conductivity endows conjugated materials with various fascinating characteristics to serve as a sensing platform (Sannicolò et al., 2014).

A major advance in the last few years is the discovery of graphene, defined as a single layer of carbon atoms patterned in a $2 \mathrm{D}$ honeycomb network, which is the rising star of 
nanomaterials received fascinating research attention in (bio)sensors (Novoselov et al., 2012). Due to the fact that graphene surface can acquire various functionalities through the creation of different receptor units and bioactive scaffolds (Gravagnuolo et al., 2015), graphene-based enantioselective sensors have attracted widespread attention in electrochemical chiral sensing and discrimination platform for bioactive molecules such as naproxen (Guo et al., 2014), cystine (Zor et al., 2015).

Herein, a novel platform based on graphene and cyclodextrin $(r G O / \gamma-C D)$ with high sensing capability toward enantiomers thanks to their complex formation (due to the chiral selector behavior) and different molecular orientations is reported. The peculiarity of this platform stands in its enantioselective capability while operating in either electrochemical or optical detection mode. Sensing behavior of $r G O / \gamma-C D$ for Trp enantiomers, an essential amino acid that is a precursor for a variety of biologically active compounds, in addition of being an important metabolite in human and animals and consequently utilized as a pharmaceutical antidepressant agent (Baytak and Aslanoglu, 2015), was studied and demonstrated via electrochemical, photoluminescence and computational methods. The results show that Trp enantiomers exhibit different affinity towards $r G O / \gamma-C D$ and the obtained sensor gives higher sensitivity towards $L$-Trp. The results ensure that the proposed enantioselective sensor can be used as an effective tool to estimate the enantiomeric quantity of $D$ - and $L$-Trp.

Graphene-related materials can be integrated as the core of bio/sensing platforms due to their excellent abilities for direct interaction with bio/molecules, electrical conductivity, their applicability in solution and the capability to be used as highly efficient long-range quenchers (Morales-Narváez and Merkoçi, 2012). After surface modification with selective groups, antibodies, aptamers and etc. for target materials, unprecedented bio/sensing strategies can be developed (Morales-Narváez et al., 2013). Here we propose the use of a 
hybrid material such as $r G O / \gamma-C D$ that can operate in both electrical and optical biosensing with interest for enantioselective sensing of $D$ - and $L$-Trp (See Scheme 1). The operation principle of such platform and its efficiency to be used in such an important field are clarified and demonstrated through a series of spectroscopic characterizations of the involved materials, electrical and optical behaviors in addition to molecular docking responses as shown in the following sections.

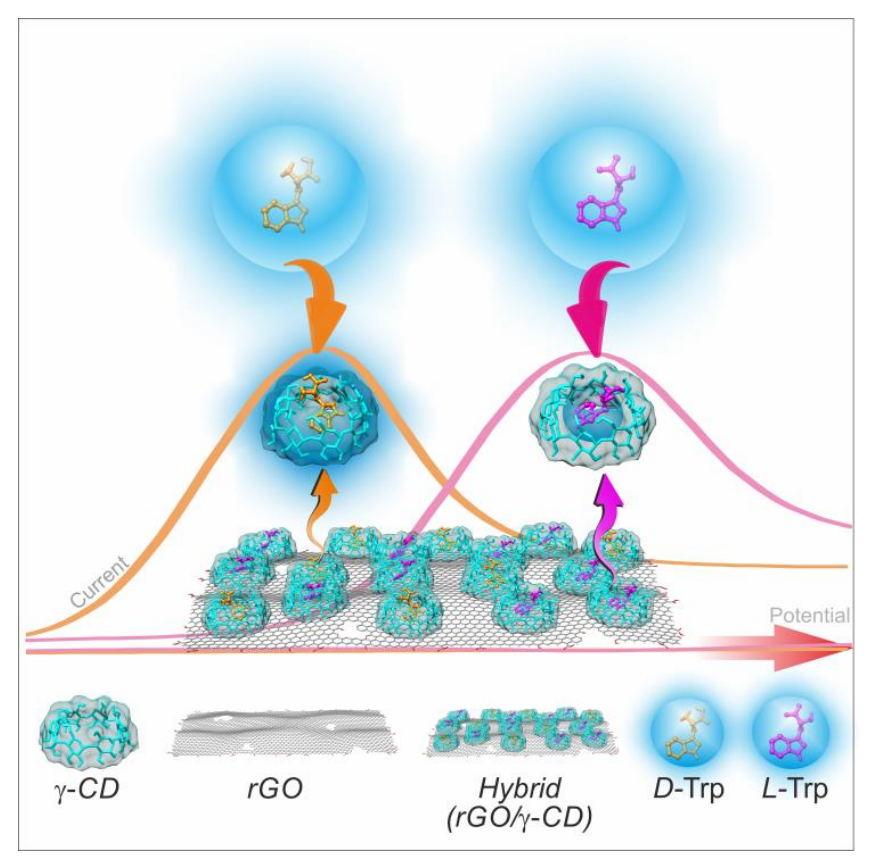

Scheme 1. Schematic, not in scale, of the graphene-based hybrid for enantioselective sensing applications operating through both optical (photoluminescent quenching) and electrochemical generated signals.

\section{Material and methods}

\subsection{Reagent and apparatus}

Graphite powder $(99.99 \%)$, concentrated $\mathrm{H}_{2} \mathrm{SO}_{4}$ and $\mathrm{H}_{3} \mathrm{PO}_{4}, \mathrm{H}_{2} \mathrm{O}_{2}(30 \%), \mathrm{KMnO}_{4}$ (99\%), $\mathrm{K}_{2} \mathrm{~S}_{2} \mathrm{O}_{8}, \mathrm{P}_{2} \mathrm{O}_{5}, \gamma$-cyclodextrin, $D$ - and $L$-Trp (tryptophan) were purchased from Sigma- 
Aldrich. All reagents were used with regard to the material safety guide recommended by the suppliers and used as received without further purification.

Transmission Electron Microscopy (TEM) images were acquired using JEOL JEM 2100F HR-TEM operating at $20 \mathrm{kV}$. AFM micrographs were obtained using a Park XE7 instrument. Fourier transformed infrared (FTIR) spectra were recorded on an FTIR spectrophotometer equipped with an attenuated total reflectance (ATR) accessory (Perkin Elmer 100 FTIR) in the $550-4000 \mathrm{~cm}^{-1}$ range in ambient air at room temperature. Thermogravimetric analysis (TGA) of the materials (10-15 mg) was performed on a Setaram thermal gravimetric analyzer at a heating ramp of $10{ }^{\circ} \mathrm{C} \min ^{-1}$ over the temperature range of 25-1200 ${ }^{\circ} \mathrm{C}$ under inert gas atmosphere. Raman spectra were recorded on a Renishaw inVia Reflex equipped with CCD detector. X-ray photoelectron spectroscopy (XPS) measurements were performed using a PHI 5000 VersaProbe analyzer/spectrometer. Photoluminescence experiments were recorded with PTI QuantaMaster ${ }^{\mathrm{TM}} 40$ steady state spectrofluorometer system (QM40) using a high intensity continuous Xe lamp. For the electrochemical measurements, a computer-controlled IVIUM-CompactStat potentiostat was used. A BAS/C3 electrochemical cell stand consisting of three electrode configuration cell was combined to potentiostat. A bare or modified GCE was used as working electrode, the reference and counter electrode were $\mathrm{Ag} / \mathrm{AgCl}$ electrode and a platinum wire, respectively. A Sonorex Super RK 106 was used to exfoliate materials and to clean electrode surfaces.

\subsection{Production of $\gamma$-cyclodextrin decorated graphene $(r G O / \gamma-C D)$ hybrid and overall} procedure

Production of $\gamma$-cyclodextrin decorated graphene $(r G O / \gamma-C D)$ hybrid and overall procedure for measurements were given as supplementary material file. 


\section{Results and discussion}

\subsection{Characterization of $r G O / \gamma-C D$ hybrid}

The structural characterization of the hybrid material was performed by FTIR, TGA, Raman spectroscopy and XPS (see Fig. S1 for XPS results). The first evidence of the successful functionalization of graphene oxide sheets was evaluated by FTIR. The spectra shown in Fig. 1a reveals that $G O$ and $r G O / \gamma-C D$ samples possess hydrophilic $\mathrm{O}-\mathrm{H}$ group (3020-3600 $\mathrm{cm}^{-1}$ ) on their surfaces, thus ensuring their good solubility in water. A clear stretching vibration of $\mathrm{CH}_{2}$ in the range of $2840-2960 \mathrm{~cm}^{-1}$ was acquired in spectra. Moreover, a stretching vibration of $\mathrm{C}=\mathrm{C}$ (aromatic) was observed for $G O, r G O$ and $r G O / \gamma$ $C D$ at 1627,1670 and $1646 \mathrm{~cm}^{-1}$, respectively. The characteristic stretching vibrational modes of $\mathrm{C}=\mathrm{O}\left(1725 \mathrm{~cm}^{-1}\right), \mathrm{C}-\mathrm{O}\left(1223 \mathrm{~cm}^{-1}\right)$ and $\mathrm{C}-\mathrm{O}\left(1047 \mathrm{~cm}^{-1}\right)$ bonds were observed in $G O$ spectrum. A comparison of the FTIR spectra of the three samples reveals two important observations. One is the disappearance of the broad $\mathrm{O}-\mathrm{H}$ band through the reduction process of $G O$ to form $r G O$, whereas an enhancement is observed due to hydroxyl groups on $\gamma-C D$ scaffold, endowing higher polarity and hydrophilicity to $r G O / \gamma-C D$. The other should be remarked with the existence of characteristic $\gamma-C D$ absorption bands at 1153 and $1029 \mathrm{~cm}^{-1}$ evidencing the settlement of $\gamma$ - $C D$ on $r G O$ sheets (Guo et al., 2010).

Further support for anchoring of $\gamma-C D$ on $r G O$ scaffold was provided by TGA analysis. Fig. $1 \mathrm{~b}$ shows the thermogravimetric profiles of $G O, r G O$ and $r G O / \gamma-C D$. Evaporation of water molecules caused a slight mass loss below $125^{\circ} \mathrm{C}$ for all samples. $G O$ remains unstable with a large mass loss $\left(69.8\right.$ wt \%) observed between 145 and $230{ }^{\circ} \mathrm{C}$ which could be ascribed to the removal of oxygen-containing functional groups on the scaffold, whereas $r G O$ is thermally more stable with a slight mass loss $(4.2 \mathrm{wt} \%)$ under the same experimental conditions. On the contrary, $r G O / \gamma-C D$ displays a significant mass loss $(30.3 \mathrm{wt}$ 
$\%$ ) between $220-450{ }^{\circ} \mathrm{C}$ which could be ascribed to the residual oxygen-containing functional groups and the decomposition of $\gamma$-CD anchored on $r G O$.
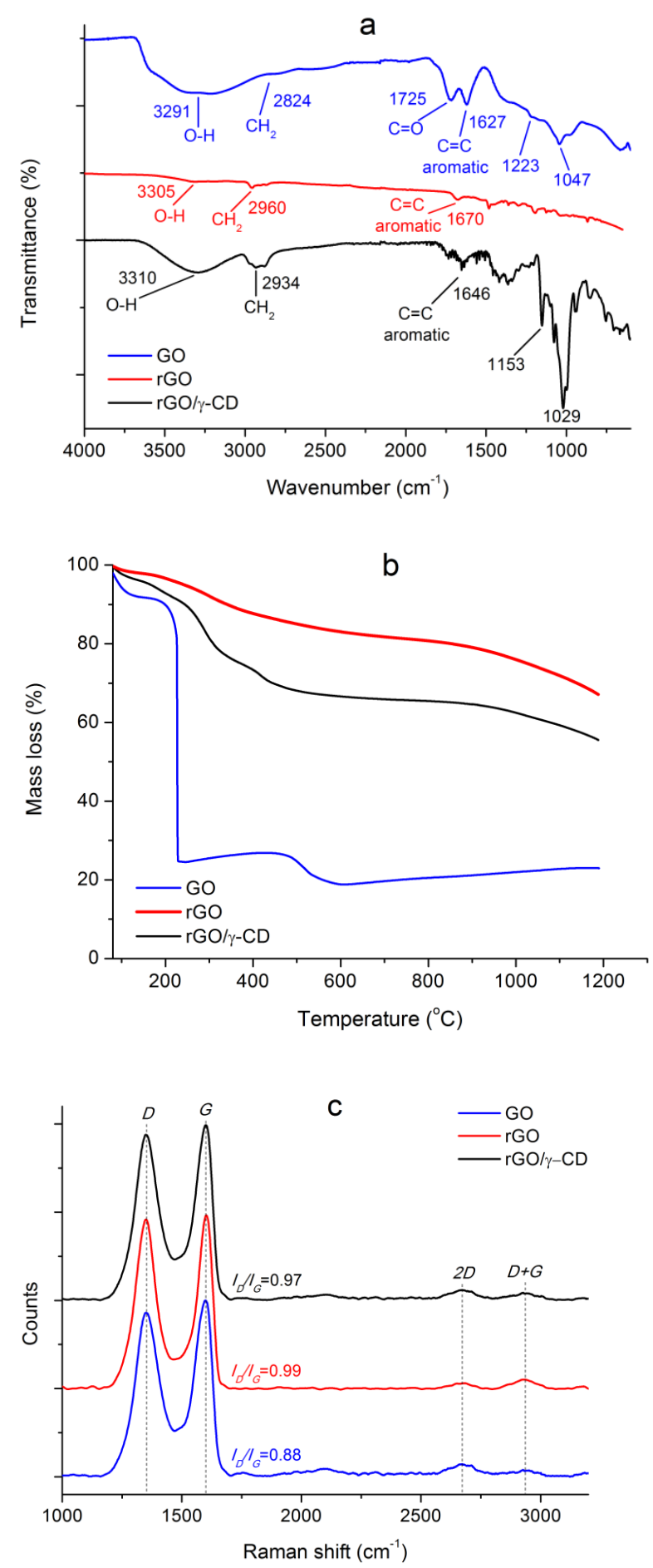

Fig. 1. FT-IR spectra (a), TGA curves (b) and Raman spectra (c) of $G O, r G O$ and $r G O / \gamma-C D$. 
Raman spectroscopy analysis was carried out to define the structural changes (disorders) after the reduction and modification processes and to inspect number of graphene layers which is one of the most important parameters for graphene-related materials. Fig. 1c shows the characteristics D $\left(1349 \mathrm{~cm}^{-1}\right), G\left(1599 \mathrm{~cm}^{-1}\right), 2 \mathrm{D}\left(2683 \mathrm{~cm}^{-1}\right)$ and D+G $\left(2935 \mathrm{~cm}^{-}\right.$ 1) Raman bands of $G O, r G O$ and $r G O / \gamma-C D$. D and $\mathrm{G}$ bands are respectively associated with the $\mathrm{sp}^{3}$ disorders in hexagonal lattice caused by oxidation and the vibration of $\mathrm{sp}^{2}$ bonded carbon atoms in the hexagonal lattice. The calculated $I_{D} / I_{G}$ ratios are $0.88,0.99$ and 0.97 for $G O, r G O$ and $r G O / \gamma-C D$, respectively, expressing that the reduction process results in disorders in hexagonal lattice but attachment of $\gamma-C D$ prevents some oxygen containing functional groups on basal scaffold of graphene sheets (Guo et al., 2010). In all spectra, the classic $2 \mathrm{D}$ bands referring to the stacking of carbon layers in graphene-related materials at $2683 \mathrm{~cm}^{-1}$ displaying wide peak characteristics were observed. For a single-layer graphene sheet, 2D band generally appears as a Lorentzian peak centered at $2679 \mathrm{~cm}^{-1}$ (Graf et al., 2007). However, as the number of layers increases, the intensity of $2 \mathrm{D}$ peak decreases by displaying a wide peak characteristic with a shift to higher wavenumbers (Wei et al., 2016). Considering these previous reports, we can conclude that our Raman results are consistent with the characteristics of multilayer graphene flakes.

The microscopic characterization was acquired with HR-TEM and AFM techniques. Subsequent to synthesis, applied drying process in petri dishes resulted in brown colored and easily scraped film-like $G O$, black granule-like $r G O$, and black hardly scraped $r G O / \gamma-C D$ materials, see Fig. 2a-c. The discrepancy between $r G O$ and $r G O / \gamma-C D$ should be remarked by intercalation of $\gamma$-CD between $\mathrm{rGO}$ sheets that prevents $r G O$ to aggregate facilitating an advantage for the electrochemical and optical application steps. 

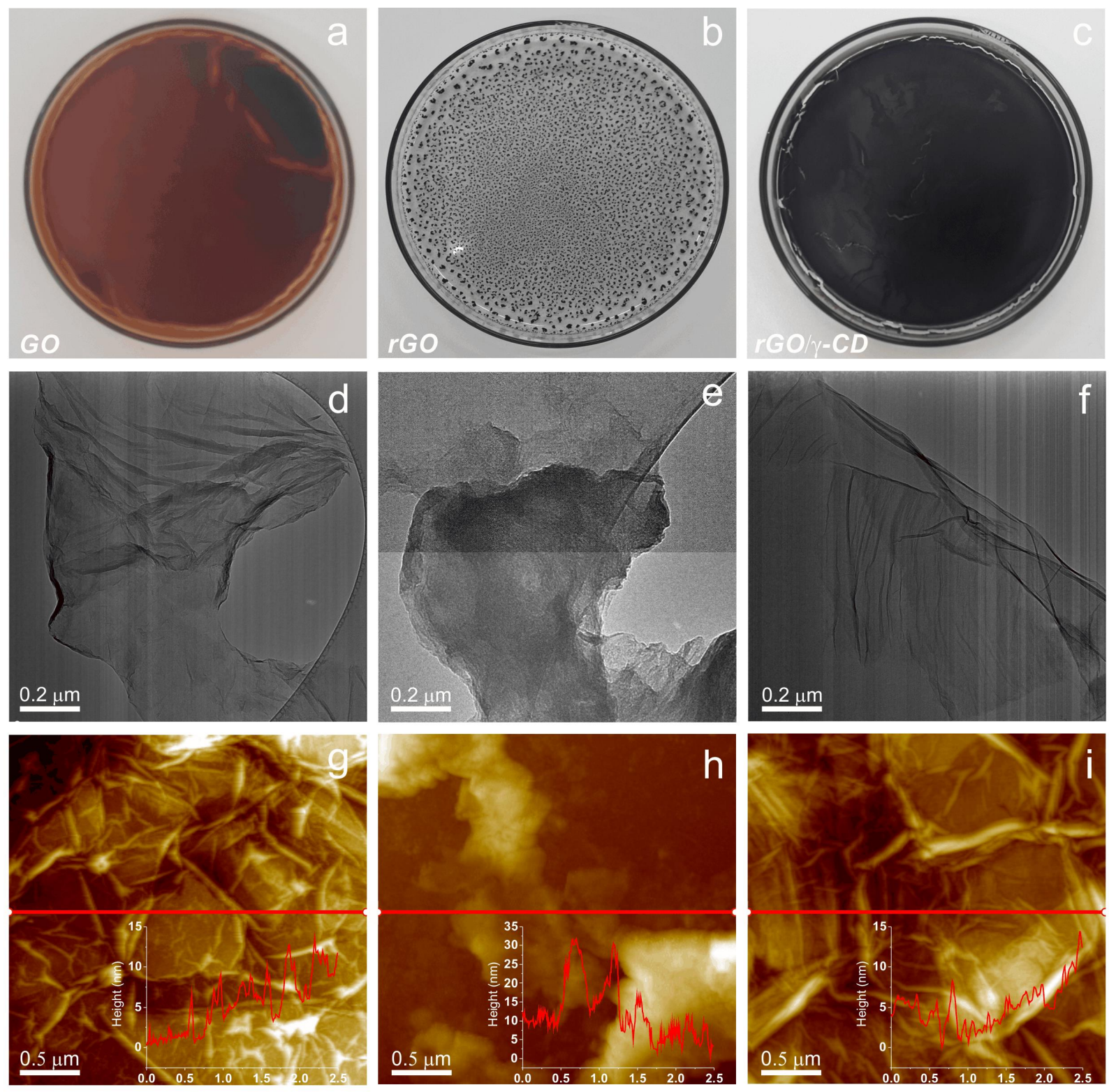

Fig. 2. $G O$ (a), $r G O$ (b), and $r G O / \gamma$-CD (c) materials dried in petri dishes. TEM and AFM micrographs of $G O(\mathrm{~d} / \mathrm{g}), r G O(\mathrm{e} / \mathrm{h})$ and $r G O / \gamma-C D(\mathrm{f} / \mathrm{i})$, respectively.

Aiming at estimating the morphology of $G O, r G O$ and $r G O / \gamma-C D$, the materials casted on the substrate were examined by TEM and AFM. The soft nature of $G O$ is readily apparent from the presence of wrinkles and overlaps (Peng et al., 2015), see Fig. 2d/g. As seen in Fig. $2 \mathrm{e} / \mathrm{h}$, the image of rGO reveals that graphene sheets are agglomerated because of the removal of oxygen-containing functional groups, leading to hydrophobic forces (Park and Ruoff, 2009). Fig. 2f/i shows the TEM and AFM images of $r G O / \gamma-C D$. Unlike rGO, a flake-like 
wrinkled topology was obviously observed, revealing the feasibility of the present strategy for surface modification step (Guo et al., 2010). On the basis of the height profiles of the inspected sections in AFM micrographs, the average thickness values of $G O, r G O$ and $r G O / \gamma$ $C D$ are observed as 5-8 $\mathrm{nm}, 16-23 \mathrm{~nm}$ and 6-9 $\mathrm{nm}$, respectively. In consistent with the Raman spectra, our AFM results verify the presence of multilayer graphene flakes, taking into account of the characteristic thickness value of single layer flake (1-2 nm) (Rao et al., 2009).

\subsection{Enantioselective response of the proposed sensor for D-and L-Trp Enantiomers}

It is well-known that the nature of binding varies for each enantiomer because how it will interact with a chiral neighbor is geometry dependent, and graphene-related materials endow large surface areas for assembling of various compounds. Herein, the enantioselective profile of $r G O / \gamma-C D$ was assessed by electrochemical and photoluminescent methods. After surface characterization of the proposed sensing platform using cyclic voltammetry $(\mathrm{CV})$ and electrochemical impedance spectroscopy (see Fig. S3 and Table S1), in order to assess the enantioselective behavior of $r G O / \gamma-C D / G C E$ for $D$ - and $L$-Trp enantiomers, CV and DPV techniques were employed. In CV measurements, the bare GCE displayed only one irreversible peak at $0.90 \mathrm{~V}$ (also $r G O / G C E$ at $0.86 \mathrm{~V}$ ) for each enantiomers ( $D$ - and $L$-Trp), depicting no discrimination of the enantiomeric pairs was established in the polarization window, see inset of Fig. 3a. As it can be clearly seen in Fig. 3a, that modification of $r G O / \gamma$ $C D$ on GCE surface led to an electrochemical transducing system upon analyte recognition and triggered well-defined irreversible anodic peaks at 0.66 and $0.84 \mathrm{~V}$ for $D$ - and $L$-Trp enantiomers, respectively. 

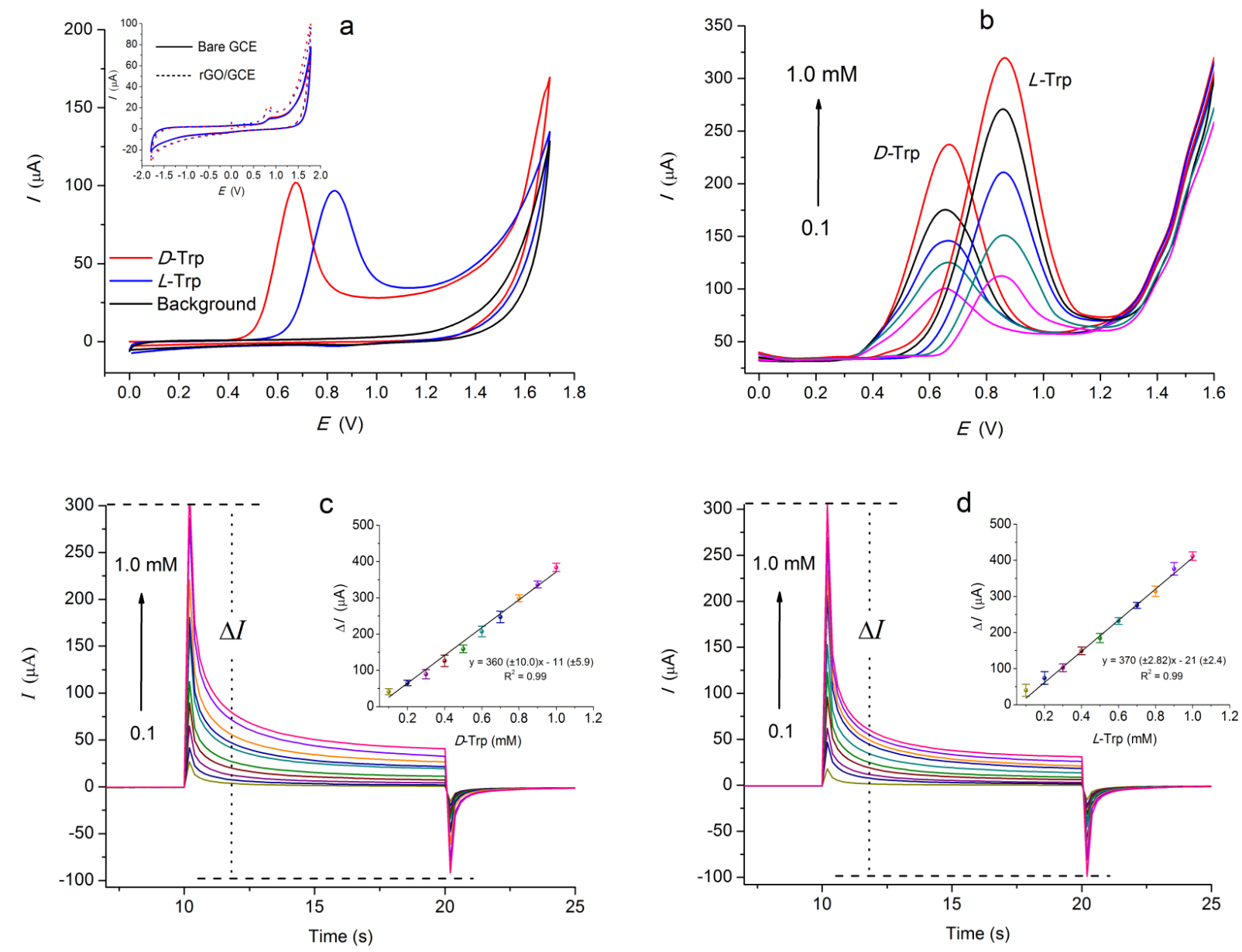

Fig. 3. Cyclic voltammograms in the absence and presence of $D$ - and $L$-Trp in A-PBS solution at $r G O / \gamma-C D / G C E$ (a). The inset is cyclic voltammograms of $D$ - and $L$-Trp at bare GCE (solid lines) and rGO/GCE (dotted lines). Differential pulse voltammograms of $D$ - and $L$-Trp upon increasing concentration of $D$-Trp and $L$-Trp in low concentration range (0.1-1.0 $\mathrm{mM})$ (b). Chronoamperograms upon increasing concentration of $D$ - $\operatorname{Trp}(\mathrm{c})$ and $L$ - $\operatorname{Trp}(\mathrm{d})(0.1-$ $1.0 \mathrm{mM})$ at $r G O / \gamma-C D / G C E$. The applied potentials are 0.00 and $0.66 \mathrm{~V}$ for $D$-Trp, 0.00 and $0.84 \mathrm{~V} v s . \mathrm{Ag} / \mathrm{AgCl}(3 \mathrm{M} \mathrm{KCl})$ for $L$-Trp. The insets show the linear correlation between the concentration and the measured peak intensity.

Considering $\mathrm{CV}$ results, $r G O / \gamma-C D$ nanocomposite presents significantly different enantioselective electrochemical response compared to bare GCE by combining the 
fascinating electronic capability of $r G O$ and the high enantioselectivity of $\gamma-C D$. It is important to note that unlike the published researches (Feng et al., 2014; Guo et al., 2013; Zhang et al., 2013) in which the authors have investigated the peak current of a redox couple, we directly investigated the peak current and potential of $D$ - and $L$-Trp without the need of any indicator which would made the system more complex in addition of increasing uncertainty in understanding the whole process. Beside Trp enantiomers, while a response was observed for arginine, mandelic acid and methionine enantiomers at the same peak potential value, no discriminative sensing capability was acquired for the other tested enantiomeric pairs (see Table S2). Aiming at examining the optimum experimental conditions, effects of $\mathrm{pH}$ and amount of modification layer $(r G O / \gamma-C D)$ on current were evaluated and change of current upon increasing concentration of $D / L$-Trp was examined by CV and DPV (see Fig. S4-6). In accordance with CV results, well-defined strong irreversible anodic peaks were acquired at different peak potential values of 0.65 and $0.86 \mathrm{~V}$ for $D$ - and $L$ Trp in DPV, respectively. A linear correlation was found between the measured peak intensity and concentration of $D$ - and $L$-Trp between 0.1 and $1.0 \mathrm{mM}$, see Fig. $3 \mathrm{~b}$.

Aiming at evaluating the performance of this sensor of $r G O / \gamma-C D / G C E$ for racemic mixtures, the enantiomeric purity of $\operatorname{Trp}$ with various concentration ratios $(1: 0,1: 1,0: 1 ; D$ - to $L$-Trp) was examined by CV, see Fig. S7. A well-defined oxidation peak was observed at the mean value $(0.77 \mathrm{~V})$ of $D$ - and $L$-Trp oxidation peak potentials depicting that a racemic mixture is involved in the medium. It is important to remark that this graphene assembled enantiosensing platform enables an opportunity not only for enantiomeric discrimination of Trp pairs, but also for estimating the enantiomeric percentage of racemic mixture in a medium. The amperometric response of the modified electrode upon increasing the concentration of $D$ - and $L$-Trp from 0.1 to $1.0 \mathrm{mM}$ was examined by CA. Fig. $3 \mathrm{c}$ and $3 \mathrm{~d}$ show that the peak current intensity for $D / L$-Trp enantiomers increases with the increasing 
concentration with a $\Delta I_{\max }$ of $386.86 \mu \mathrm{A}$ and $412.44 \mu \mathrm{A}$, respectively. A linear correlation was found between the concentration and the measured peak intensity (Insets of Fig. 3c and 3d). The sensitivities of this sensor for $D$ - and $L$-Trp were $360 \mu \mathrm{AmM}^{-1}$ and $370 \mu \mathrm{AmM}^{-1}$, and the limit of detection (LOD) were calculated as $54 \mu \mathrm{M}$ and $21 \mu \mathrm{M}$ using the standard deviation of $y$-intercept and the slope of the regression line. Considering the results and the calculations, the proposed sensor displays higher sensitivity with a lower detection limit towards $L$-Trp. The specific discrimination features of the proposed sensor were compared to other GCE-based electrochemical sensors reported for chiral recognition of Trp enantiomers and the results were shown in Table S2. The proposed sensor has advantageous over the compared ones by endowing recognition difference not only with the increase in peak current but also with the discrepancy in peak potential. In addition, it offers a reasonable linear range for Trp enantiomers and the limit of detection values are lower than the compared reports (Table S3).

The interaction between Trp enantiomers and $r G O / \gamma-C D$ in aqueous media can be also followed through photoluminescence measurements. Fig. $4 \mathrm{a}$ and $4 \mathrm{~b}$ present the photoluminescence spectra change of Trp enantiomers upon addition of $r G O / \gamma-C D(0.0-36.0$ $\left.\mu \mathrm{g} \mathrm{mL}^{-1}\right)$. On the basis of change in the emission spectra $r G O / \gamma-C D$ quenching capability mainly toward $L$-Trp rather than for $D$-Trp, we could assess that $r G O / \gamma$ - $C D$ displayed higher affinity for $L$-Trp. The photoluminescence quenching levels were explored varying the quantity of $r G O / \gamma$-CD. As expected, the photoluminescence intensity of $L$-Trp displayed a decrease sequentially and almost quenched upon addition of the specified quantity of $r G O / \gamma$ $C D$, whereas only decreased for $D$-Trp. Importantly, it is well-known that the energy transfer phenomenon exhibits a dependence on spatial orientation of the dipoles of the studied acceptor and donor (Ji et al., 2015; Morales-Narváez et al., 2012). Consequently, it is likely that the spatial orientation between the complex $r G O / \gamma-C D$ and the explored enantiomers is 
respectively different, leading to a different photoluminescence quenching modulation proportionately. A linear correlation was found between the quantity of $r G O / \gamma-C D(0.0-36 \mu \mathrm{g}$ $\mathrm{mL}^{-1}$ ) and the peak intensity (Insets of Fig. $4 \mathrm{a}$ and $4 \mathrm{~b}$ ). Interestingly, it should be noted that no quenching of Trp enantiomers upon addition of $\gamma-C D$ was observed, and a negligibly small decrease was acquired using $G O$ and $r G O$ for the tested experimental conditions. The result could be ascribed to unification of the discriminative sensing capability of $\gamma$ - $C D$ (Tao et al., 2014) anchored on $r G O$ sheet and quenching character of $r G O$ (Morales-Narváez et al., 2015).
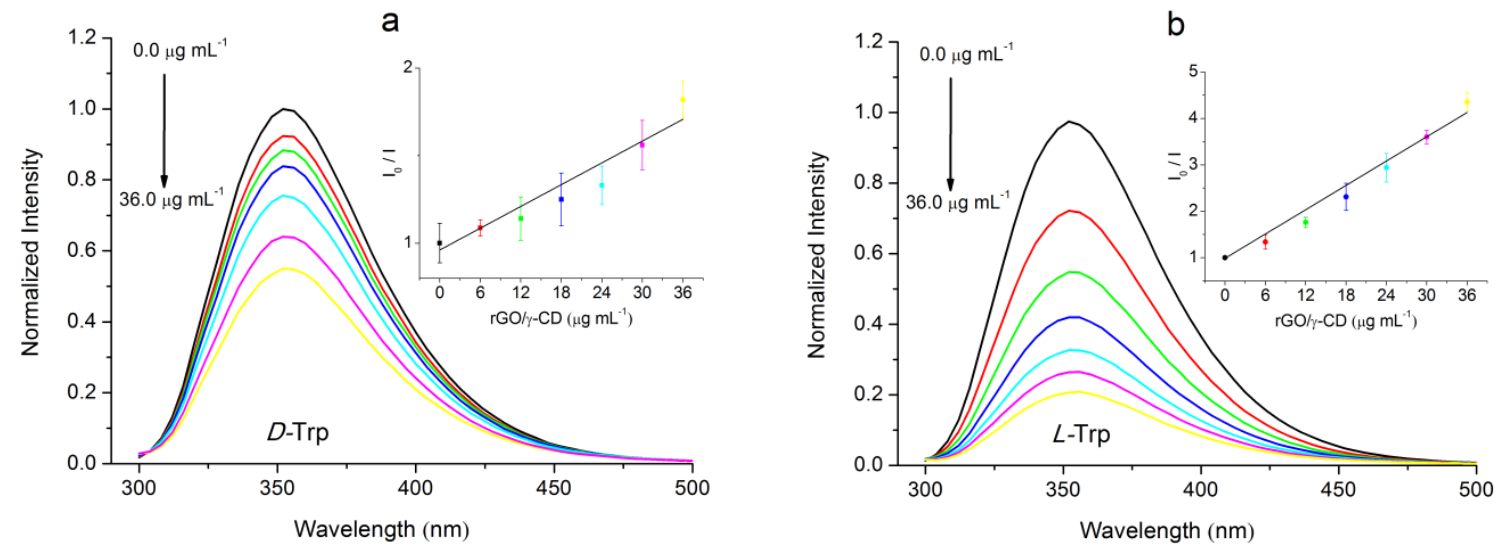

Fig. 4. The change in photoluminescence intensity of $D$ - (a) and $L$-Trp (b) in aqueous media upon addition of $r G O / \gamma-C D\left(0.0-36.0 \mu \mathrm{g} \mathrm{mL}^{-1}\right)$. The insets show the linear correlation curves for $D$ - and $L$-Trp.

Understanding the essence of this enantioselective behavior in molecular level is of crucial importance. Modeling of molecular interactions should provide deeper insights to assess the shape and size complementarities and to evidence experimental results. Herein, considering the advantage of the mentioned research, molecular docking studies were carried 
out by employing ADV in two steps; (i) Establishing the settling conformational structure of $\gamma-C D$ on $G O$. As we have recently reported, $\alpha-C D$ and $\beta-C D$ favorably assemble on the basal scaffold of $G O$ from their distinctly hydrophilic wider rims (Zor et al., 2015, 2014). As expected in this sense, $\gamma-C D$ assembled on $G O$ scaffold from its wider rim by hydrogen bonding and also electrostatic interactions (Fig. 5a). Gibbs free energy $(\Delta \mathrm{G})$ for the interaction between $\gamma-C D$ and $G O$ was estimated as $-9.8 \mathrm{kcal} \mathrm{mol}^{-1}$ in this molecular docking step. Most of the oxygen containing groups on basal scaffold of $G O$ were removed by reducing process, thereby the resultant could be presented as shown in Fig. 5b. (ii) Investigation of complexation mechanism between $r G O / \gamma-C D$ and Trp enantiomers. As seen from conformational sampling in Fig. $5 \mathrm{c}$ and $5 \mathrm{~d}$, the cavity size of the $\gamma-C D$ is large enough to allow the Trp enantiomers to penetrate by keeping them inside the cavity via a high stability constant. Interestingly, chiral character of Trp enantiomers and $\gamma-C D$ implemented significant conformational alterations in docking modes upon complexion. Gibbs free energies representing the strength of the interaction between $\gamma-C D$ and $D-/ L$-Trp were estimated as $-5.8 \mathrm{kcal} \mathrm{mol}^{-1}$ and $-6.0 \mathrm{kcal} \mathrm{mol}^{-1}$, respectively. It should be noted that this discrepancy in Gibbs free energy gives rise to a significant difference in the binding constants of $D$ - and $L$ Trp estimated as $1.79 \times 10^{4}$ and $2.50 \times 10^{4} \mathrm{M}^{-1}$, respectively. Besides $D$ - and $L$-Trp, the molecular docking was employed to evaluate Gibbs free energies and binding constants of the other tested chiral compounds (arginine, mandelic acid and methionine, which gave a signal in electrochemical measurements with no discriminative sensing, in light of the interaction strength) and the results were given in Table S4. Gibbs free energies were calculated as -5.3 $\mathrm{kcal} \mathrm{mol}{ }^{-1},-5.6 \mathrm{kcal} \mathrm{mol}^{-1}$ and $-4.3 \mathrm{kcal} \mathrm{mol}^{-1}$ and binding constants were determined as $7.71 \times 10^{3}, 1.28 \times 10^{3}, 1.43 \times 10^{3} \mathrm{M}^{-1}$ for arginine, mandelic acid and methionine, respectively. As noticed, interestingly, no discrepancy in Gibbs free energies and thus in binding constants was observed for the interaction of enantiomeric pairs of the employed chiral compounds with 
$r G O / \gamma-C D$ which proves the experimental results. Herein, it is important to remark that the cavity of $\gamma-C D$, which is hydrophobic to undertake such interactions and large enough for Trp to fit in, plays a key role in conformational alterations of docking modes upon complexion, which was observed also in the previously published study (Wei et al. 2010).
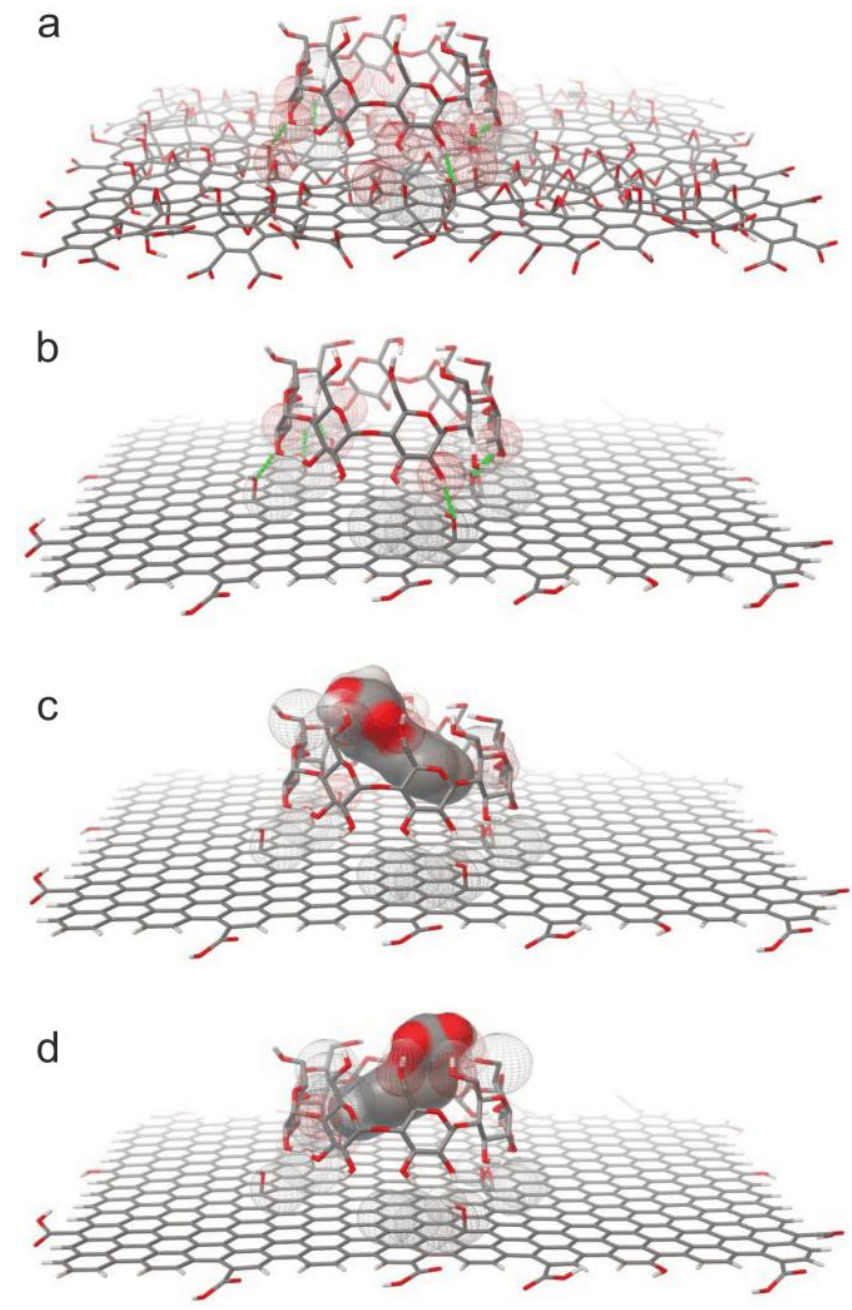

Fig. 5. The structures of $G O / \gamma-C D$ (a) and $\mathrm{rGO} / \gamma-C D$ (b) after molecular docking process. The conformational sampling representation for the interaction of $r G O / \gamma-C D$ with $D$-Trp (c) and $L-\operatorname{Trp}(\mathrm{d})$

Comparing the aforementioned results above with other chiral measurement methods for discriminative/enantioselective sensing of tryptophan enantiomers, the proposed method is shown to be more valuable because of various advantages such as less time consuming, no 
need for expensive instrumentation and reagents, and less complicated. For example, Wei et al. have investigated chiral discrimination of $D$ - and $L$-Trp by the observation of fluorescence lifetime differences of enantiomers based on the addition of BSA and CD (Wei et al., 2010). In accordance with our results, they have reported that BSA and $\gamma$-CD can provide a better chiral microenvironment for Trp enantiomers, whereas the method is rather complicated because of employing pyrene, tryptophan and bovine serum albumin (or cyclodextrin) to form ternary complexes. Another study reported by Zhou et al., in which high performance liquid chromatography (HPLC) coupled with chemiluminescence (CL) detection was employed for the separation of tryptophan (Trp) enantiomers, requires expensive columns system and highcost equipment. Considering these previous reports, it can be concluded that our method is simpler, of lower cost and with satisfying results for discriminative sensing of $\operatorname{Trp}$ enantiomers.

\section{Conclusions}

From the results mentioned above, we can conclude that $r G O / \gamma-C D$ is an effective material which can be easily applied as receptor in solid phase onto the surface of an electrochemical transducer (electrode) for discrimination of $D$ - and $L$-Trp enantiomers and/or as optical receptor dispersed in solution for enantioselective recognition of $D$ - and $L$-Trp enantiomers. This manuscript demonstrates for the first time the use of photoluminescence quenching capabilities of this kind of hybrid material for enantiosensing applications of chiral molecules which are crucial challenge in biological phenomena. Moreover, a combination of energetic profiles (molecular docking studies) with experimental results can be considered as a novel tool which allows more rational explanations for discrimination of various chiral analytes and the approach may have broad interest in the sensing field. A drawback of the proposed sensor is that it only allows sensing of racemic mixture of $D$ - and $L$-Trp 
enantiomers, without being able to evaluate the enantiomeric excess, which in addition to practical applications will be object of future research.

\section{Acknowledgements}

This work was supported by TÜBİTAK (113Z664 and BİDEB-2214-A/1059B141400210) and was produced from a part of E. Zor's PhD thesis. Nanobiosensors and Bioelectronics Group acknowledges the support from MINECO (project MAT2014-52485-P and Severo

Ochoa Program, Grant SEV-2013-0295) and Secretaria d'Universitats i Recerca del Departament d'Economia i Coneixement de la Generalitat de Catalunya (2014 SGR 260).

\section{Appendix A. Supplementary material}

Supplementary data associated with this article can be found in the online version. 


\section{References}

Alarcón-Angeles, G., Pérez-López, B., Palomar-Pardave, M., Ramírez-Silva, M.T., Alegret, S., Merkoçi, A., 2008. Carbon N. Y. 46, 898-906.

Baytak, A.K., Aslanoglu, M., 2015. Sensors Actuators B Chem. 220, 1161-1168.

Bobbitt, D.R., Linder, S.W., 2001. TrAC Trends Anal. Chem. 20, 111-123.

Cao, H., Zhu, X., Liu, M., 2013. Angew. Chemie Int. Ed. 52, 4122-4126.

Ema, T., Tanida, D., Sakai, T., 2007. J. Am. Chem. Soc. 129, 10591-10596.

Feng, W.L., Liu, C., Lu, S.Y., Zhang, C.Y., Zhu, X.H., Liang, Y., Nan, J.M., 2014. Microchim. Acta 181, 501-509.

Graf, D., Molitor, F., Ensslin, K., Stampfer, C., Jungen, A., Hierold, C., Wirtz, L., 2007. Nano Lett. 7, 238-242.

Gravagnuolo, A.M., Morales-Narváez, E., Matos, C.R.S., Longobardi, S., Giardina, P., Merkoçi, A., 2015. Adv. Funct. Mater. 25, 6084-6092.

Guo, L., Huang, Y., Zhang, Q., Chen, C., Guo, D., Chen, Y., Fu, Y., 2014. J. Electrochem. Soc. 161, B70-B74.

Guo, L., Zhang, Q., Huang, Y., Han, Q., Wang, Y., Fu, Y., 2013. Bioelectrochemistry 94, 8793.

Guo, Y., Guo, S., Ren, J., Zhai, Y., Dong, S., Wang, E., 2010. ACS Nano 4, 4001-4010.

Ji, X., Wang, W., Mattoussi, H., 2016. Nano Today. 11, 98-121.

Kaminker, R., de Hatten, X., Lahav, M., Lupo, F., Gulino, A., Evmenenko, G., Dutta, P., Browne, C., Nitschke, J.R., van der Boom, M.E., 2013. J. Am. Chem. Soc. 135, 1705217059.

Morales-Narváez, E., Hassan, A.-R., Merkoçi, A., 2013. Angew. Chemie Int. Ed. 52, 1377913783.

Morales-Narváez, E., Merkoçi, A., 2012. Adv. Mater. 24, 3298-3308.

Morales-Narváez, E., Naghdi, T., Zor, E., Merkoçi, A., 2015. Anal. Chem. 87, 8573-8577.

Morales-Narváez, E., Pérez-López, B., Pires, L.B., Merkoçi, A., 2012. Carbon N. Y. 50, 2987-2993.

Novoselov, K.S., Fal'ko, V.I., Colombo, L., Gellert, P.R., Schwab, M.G., Kim, K., 2012. Nature 490, 192-200.

Park, S., Ruoff, R.S., 2009. Nat. Nanotechnol. 4, 217-224.

Peng, L., Xu, Z., Liu, Z., Wei, Y., Sun, H., Li, Z., Zhao, X., Gao, C., 2015. Nat. Commun. 6, 5716.

Rao, C.N.R., Sood, A.K., Subrahmanyam, K.S., Govindaraj, A., 2009. Angew. Chem. Int. Ed. Engl. 48, 7752-7777. 
Sannicolò, F., Arnaboldi, S., Benincori, T., Bonometti, V., Cirilli, R., Dunsch, L., Kutner, W., Longhi, G., Mussini, P.R., Panigati, M., Pierini, M., Rizzo, S., 2014. Angew. Chemie 53, $2623-2627$.

Tao, Y., Dai, J., Kong, Y., Sha, Y., 2014. Anal. Chem. 86, 2633-2639.

Trojanowicz, M., 2014. Electrochem. commun. 38, 47-52.

Wang, L., Zhang, Z.J., Huang, L.G., 2008. Anal. Bioanal. Chem. 390, 1431-1436.

Wattanakit, C., Côme, Y.B. Saint, Lapeyre, V., Bopp, P. a, Heim, M., Yadnum, S., Nokbin, S., Warakulwit, C., Limtrakul, J., Kuhn, A., 2014. Nat. Commun. 5, 3325.

Wei, X., Meng, Z., Ruiz, L., Xia, W., Lee, C., Kysar, J.W., Hone, J.C., Keten, S., Espinosa, H.D., 2016. ACS Nano 10, 1820-1828.

Wei, Y., Wang, S., Shuang, S., Dong, C., 2010. Talanta 81, 1800-1805.

Zhang, Q., Guo, L., Huang, Y., Wang, Y., Han, Q., Fu, Y., 2013. Anal. Methods 5, 43974401.

Zhou, J., Chen, S., Sun, F., Luo, P., Du, Q., Zhao, S., 2015. J. Chromatogr. B Anal. Technol. Biomed. Life Sci., 1006, 65-70.

Zor, E., Bingol, H., Ramanaviciene, A., Ramanavicius, A., Ersoz, M., 2015. Analyst 140, 313-321.

Zor, E., Esad Saglam, M., Alpaydin, S., Bingol, H., 2014. Anal. Methods 6, 6522-6530.

Zor, E., Hatay Patir, I., Bingol, H., Ersoz, M., 2013. Biosens. Bioelectron. 42, 321-325. 


\section{Graphical abstract}

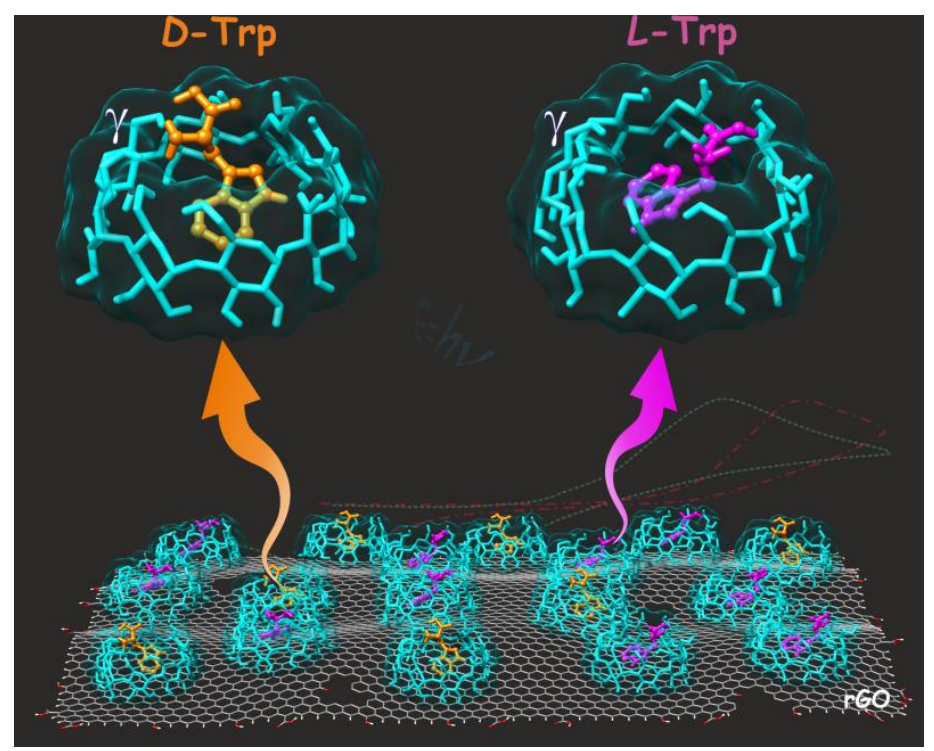

\section{Research Highlights}

- A graphene/cyclodextrin $(r G O / \gamma-C D)$ hybrid for distinguishing between different chiral forms of a compound was proposed.

- The enantioselective profile of $r G O / \gamma-C D$ was assessed by electrochemical techniques, photoluminescent and computational methods.

- The combination of energetic profiles with experimental results can be considered as a novel aspect which allows more rational explanations for discrimination of various chiral analytes in sensor area. 